\title{
E-SERVICES INTEROPERABILITY ANALYSIS AND ROADMAP ACTIONS
}

\author{
Aphrodite Tsalgatidou, Eleni Koutrouli \\ Department of Informatics \& Telecommunications, National \& Kapodistrian University of \\ Athens, Greece \\ \{atsalga,ekou\}@di.uoa.gr
}

\begin{abstract}
e-Services are the building blocks for loosely-coupled, distributed applications based on the Service Oriented Architecture's principles. One of the major benefits they offer is interoperability both between components of Service Oriented systems and between different systems. Still, the variety and diversity of implementations and interpretations of SOA and the vast amount of emerging standards hinder interoperability. This paper examines the interoperability requirements and related issues for each one of the major $e-$ Services categories: Web, Grid and P2P services. Our aim is to provide the basis for a roadmap towards improving interoperability of e-Services which will enable the successful formation of service-based distributed applications.
\end{abstract}

\section{INTRODUCTION}

Service Oriented Computing has revolutionized the way Information Technology is structured and has stimulated great expectation among the developers community. Service Oriented Architectures (SOA) emerged with the promise to support the loose coupling of system parts in a far better way than existing technologies and provide agility, flexibility and cost savings via reusability, efficiency and interoperability both between components of Service Oriented systems and between different systems.

However, the lack of agreement on what constitutes a SOA and the vast amount of emerging standards makes it difficult to understand and utilize the potentials of eServices technologies. In this context, interoperability, which is one of the basic characteristics and benefits of SOA, needs to be further explored in order to find out the open issues and best practices.

In this paper we present the interoperability requirements and related issues in the e-Service area, considering interoperability both in terms of intra- and interparadigm integration. Our goal is to pinpoint the challenges and provide a roadmap of best practices for interoperability.

The rest of the paper is organised as follows: In section 2 we examine the major trends in Service Oriented technology which lie in the areas of Web, P2P and Grid services, with a focus on the standardization efforts in each area. We also provide the interoperability issues in each one of the three areas, synergies and integration efforts, and we present roadmap actions for intra- and inter- paradigm interoperability. In section 3 we provide our concluding remarks and a summary of 
the main interoperability issues, the current approaches and trends towards interoperability as well as roadmap actions for interoperability in a table that provides a general interoperability overview for the three areas of e-Services.

\section{E-SERVICES AND INTEROPERABILITY}

According to $\mathrm{W} 3 \mathrm{C}$ (W3C, 2005), a Service Oriented Architecture (SOA) specifies a set of components whose interfaces can be described, published, discovered and invoked over a network. SOA is characterized by three basic service operations: publishing, discovery and invoking. Value added operations like composition and management can also be provided. Web Services (WS), Grid and P2P Services adhere to the SOA model and are all known as e-Services.

In the e-Services domain we view interoperability as the ability of systems, applications and services, to communicate, exchange data and files, work together or operate on behalf of one another. Standardization is one of the basic ways to achieve e-Services interoperability, therefore, in the following we briefly describe the three e-Services categories with a focus on standardization efforts in each area.

"A Web Service is a software application identified by a URI, whose interfaces and binding are capable of being defined, described, and discovered by XML artifacts, and supports direct interactions with other software applications using XML-based messages via Internet-based protocols" (W3C, 2002). What makes the Web Service vision attractive is the ability to discover the Web Services that fulfill users' needs, negotiate service contracts and have the services delivered where and when the users need them (Tsalgatidou, 2002). We are currently witnessing the rapid development and maturation of a stack of interrelated standards that are defining the Web Service infrastructure along with a great number of development tools that support the Web Service development. The key standards for describing, advertising, discovering and binding WSs are WSDL (WSDL, 2001) UDDI (UDDI, 2005) and SOAP (SOAP, 2001). Besides, there are ongoing standardization efforts in WS composition, orchestration, choreography, security and management, e.g. BPEL4WS (BPEL4WS, 2005), ebXML (ebXML, 2005), WS-Security (WSSecurity, 2002), etc. An overview of the various WS standards that compose the current WS protocol stack, along with details regarding their standardization status can be found in (Wilkes, 2005).

The term Grid refers to "a system that is concerned with the integration, virtualization, and management of services and resources in a distributed, heterogeneous environment that supports collections of users and resources (virtual organizations) across traditional administrative and organizational domains (real organizations)" (OGSA Glossary, 2005). Significant effort led by the Global Grid Forum (GGF, 2005) has been channelled toward the standardization of protocols and interfaces. Such en effort is the Open Grid Services Architecture Grid Architecture (OGSA) (Foster, 2002) which integrates Grid and Web Services. OGSA was initially materialized by the Open Grid Services Infrastructure (OGSI) (OGSI, 2003), and more recently by the Web Services Resource Framework (WSRF) proposal (WSRF, 2004) which together with Web Services Notification specification (WS-Notification, 2004) have been submitted to OASIS (OASIS, 2005) for standardization. Many Grid communities use the open source Globus 
Toolkit (Globus, 2005)(Foster, 1999) as a technology base. Currently, the efforts of the major industry players are also targeted to the support of Globus.

The term "Peer-to-Peer" (P2P) refers to a class of systems and applications that takes advantage of resources - storage, cycles, content, human presence - available at the edges of the Internet. P2P systems cover a large spectrum of application domains such as distributed computing, file sharing, collaboration, and platforms. Standards for P2P technologies have not yet been established. Efforts for defining specifications are made by the P2P Working Group (P2PWG, 2005) whereas two standardization initiatives relevant to P2P networking are Jabber (Jabber, 2002) and JXTA (JXTA, 2005). Jabber is an XML protocol for instant messaging applications that is currently being standardized under IETF (IETF, 2005). JXTA is an opensource framework for P2P systems, initiated by SUN that supports only a minimal underlying architecture as a base.

\subsection{Intra-Paradigm Interoperability Roadmap}

In each e-Service category interoperability has specific context. In the following we identify the specific requirements for interoperability in the areas of Web, Grid and P2P services and present the ways these interoperability concerns are being addressed by each category of e-Services. We also propose roadmap actions for intra-paradigm interoperability. Furthermore, we present synergies between different kinds of e-Services which also promote Service Oriented systems interoperability and present roadmap actions for inter- paradigm interoperability.

\section{Web Services Interoperability}

Web Services promise universal interoperability and integration by establishing commonly agreed protocols for mutually understanding what a service offers and for delivering this functionality in an implementation independent way. Interoperability of legacy applications is also enabled by allowing legacy applications to be exposed as WSs and, thus, facilitating a seamless integration between heterogeneous systems. Furthermore, new services can be created and dynamically published and discovered without disrupting the existing environment. Thus, WS technology provides a standard means of interoperating between different software applications, running on a variety of platforms and/or frameworks.

The issue of WS interoperability is addressed at a conceptual level by the W3C's Web Services Architecture (WSA) document (Booth, 2004), which identifies those global elements of the global Web Services network that are required in order to ensure interoperability between Web Services. The various WS standards and enabling technologies address technical level interoperability.

The common standards for the basic WS activities description, publication and invocation (WSDL, UDDI, SOAP) have effectively become de facto standards, and, thus, support basic technical interoperability. However, there is a need for enhanced interoperability both for basic WS activities, like description, as well as value added ones, like WS management and composition and also for non functional aspects like security. In the following, we describe the interoperability requirements concerning the aforementioned aspects. 
Web Services description languages should support, beyond the functional characteristics of WSs, the description of semantic information that will enable the proper and meaningful interoperation between different WSs or applications. Semantic description is not supported by WSDL and is partially addressed by ebXML. WS description semantics can be offered by standardized ontology languages, such as OWL (OWL, 2004) and OWL-S (OWL-S, 2004), and a richer model of services, which incorporate ontologies of action to identify the effects of actions and concepts such as contracts, institutions, roles and regulations (Booth, 2004).

Web Service management which refers to monitoring, controlling, and reporting of WS qualities and WS usage, also requires semantics, management policies and management capabilities, which should be understood by the requester and provider entities (Booth, 2004).

In order to improve the security and reliability of WSs and to address more complex business scenarios that require WSs composition, a wide range of protocols have been proposed, the most prominent of which is BPEL4WS (BPEL4WS, 2004) while others like WS-Choreography (WS-Choreography, 2002) haven't completed yet the standardization process. Furthermore, different sets of vendors have been producing competing specifications in similar areas (e.g. WS-Coordination (WSCoordination, 2004) and WS-CF (WS-CF, 2003)) making it difficult for widely accepted standards to emerge even where there is a clear need for standardization (e.g., security). Thus, developers can either build up services that do not use such capabilities or develop ad-hoc solutions that may lead to interoperability problems.

Enhancing interoperability between different implementations of WS technologies is the goal of the Web Services Interoperability (WS-I) organization (WS-I, 2005), which produces documents, like the WS-I Basic Profile and WS-I basic security profile that define how existing, stable and widely accepted WS standards should be used.

In order to address the aforementioned interoperability issues, we propose the following roadmap actions: (Similar work can be found in (Wilkes, 2005).)

1. Monitor progress of protocols through key standards bodies

2. Establish policy for compliance with standards

3. Use WS-I profiles wherever relevant. Create local profiles only where necessary, and plan to upgrade to WS-I as they are published

4. Coordinate use of protocols to ensure consistent implementation of versions and profiles. Publish best practices

5. Use ontologies and ontology languages, e.g. OWL, for provision of semantics concerning WSs activities, e.g. WS description and WS management

6. Use implementations of standards that have proven interoperability

7. Wherever possible wait for implementation of protocols in products

8. Keep up with the advancement of standards

\section{Grid Services Interoperability}

Grid services interoperability can be viewed along two different dimensions:

- interoperability between distributed resources in a Grid application, and

- interoperability between different Grid applications.

Interoperability between different distributed resources in a Grid application is a main goal of the various Grid projects despite the different infrastructures they use 
and the different aspects on which they focus. The OGSA/OGSI and WSRF models provide a framework for Service Oriented Grids aiming at supporting interoperability of distributed services and resources. Grid middleware implementations based on these models (e.g. Globus Toolkit (Globus, 2005), IGENE (Furmento, 2004)) provide services such as identification, authentication and authorization and promote interoperability by allowing interoperation of Grid components independently of the operating system and network topology. However, there are interoperability issues when different entities use the Grid for sharing purposes. Efforts are being undertaken to deal with the authorization of a community in a Grid environment like the Community Authorization Service (CAS) of Globus.

Significant effort has also been channelled toward enabling interoperability between different Grid applications. There is a number of different approaches that include the definition of a minimal set of Grid services which enable the interoperation of different Grid applications (Snelling, 2002), the integration of different Grid infrastructures (Wieder, 2002), the common Grid resources description (Brooke, 2003) and the Semantic Grid (Roure, 2005) which aims at providing interoperability across time as well as space both for anticipated and unanticipated reuse of services, information, and knowledge. Furthermore, the Enterprise Grid Alliance (EGA, 2005) has recently released a reference model for enterprise grids, laying the foundation for standardized solutions that will enable cross-departmental and cross-organizational interactions.

The above analysis of Grid services interoperability issues leads to the identification of the following roadmap actions:

1. Monitor progress of protocols through key standards bodies

2. Adapt standards that have gained broad acceptance (e.g. WS+I standards)

3. Conform to a reference model (e.g. EGA's reference model for enterprise grids)

4. Adapt semantic grid services vision

5. Use tools that have gained industry acceptance (e.g. Globus)

\section{P2P Services Interoperability}

$\mathrm{P} 2 \mathrm{P}$ services interoperability, similarly to Grid Services, can be viewed either as:

- interoperability between different peers in a P2P network, or

- interoperability between different P2P applications.

Interoperability between different peers of P2P systems needs advanced interoperability techniques, since the various member nodes of a P2P network that have variety of operating systems, networking technologies and other platforms in business applications need to communicate, exchange content and aggregate their diverse resources, such as computing power or storage space. Most P2P systems use proprietary implementations and protocols for the peers interoperation and functionality. XML is expected to support peers interoperability in P2P systems, since it can be used for messaging, data storage, application deployment, resource description and distributed search (Buehling, 2001). A uniform resource description is commonly agreed to be a basic requirement that can be addressed by ontologies and standardized ontology languages, e.g. OWL, or local schema mappings (Aberer, 2004) and semantic routing which are the basis of several recent proposals for $P 2 P$ data management, e.g. the Edutella project (Edutella, 2005) which facilitates the semantic discovery of resources based on RDF (RDF, 2005). 
Interoperability between different P2P applications has not been addressed by the early P2P applications, which set up closed peer networks, accessible only to them. A user of a file sharing P2P system like Napster can not send an instant message to a fellow user without launching an Instant Messaging client to do so. Currently, only a few P2P systems are able to interoperate, such as Avaki with Sun's Grid, and Magi with JXTA (Milojicic, 2003). Efforts towards improved interoperability are made by the P2P Working Group (P2PWG, 2005). Early attempts on interoperability include Jabber and Groove Networks (Groove, 2005) which are mainly extensible attempts, and not fully interoperable systems. A common infrastructure that will contain the core services of a P2P application and can be shared by $\mathrm{P} 2 \mathrm{P}$ applications, making them easier to develop and deploy, could be a more appropriate approach. Sun Microsystems' JXTA and Microsoft's .Net Framework (Microsoft .Net, 2005) are the most significant efforts to define the core elements of a P2P platform.

Based on the above, we propose the following roadmap actions for P2P services interoperability:

1. Agreement on the use of uniform P2P resources description, e.g. using ontologies, OWL and local schema mappings

2. Agreement on an underlying platform with core $\mathrm{P} 2 \mathrm{P}$ elements for building $\mathrm{P} 2 \mathrm{P}$ systems

3. Establishment of a collection of $\mathrm{P} 2 \mathrm{P}$ standards that could launch $\mathrm{P} 2 \mathrm{P}$ as a global way of doing business

4. Use of XML in P2P systems

\subsection{Inter-Paradigm Interoperability Roadmap}

Integration of heterogeneous e-Services allows the exploitation of the specific characteristics and benefits of each e-Services type by the other types of e-Services and the alleviation of their complexities. In the following we present synergies between Web, Grid and P2P services and propose roadmap actions for interparadigm interoperability that could lead to more flexible, efficient and interoperable Service-Oriented systems.

\section{Convergence of Different Types of e-Services}

As already discussed, Grid and WS technologies are in a convergence process, led by the OGSA/OGoSI and WSRF proposals. Grid developers can, thus, exploit the experience from the WS community and concentrate on building the higher-level services that are specific to the Grid application domain. There is a need, however, for Web Service specifications that could safely be used for building interoperable Web Service Grids, and for this reason a Web Service specification profile WS-I+ has been proposed in (Atkinson, 2004). Another proposal on how Grid applications could be built using existing WS specifications is found in (Parastatidis, 2005). An example of WSs and Grid services working together is found in the Web Security Services offered by McAffee.com which has implemented a security Grid with millions of sensors using a WS framework (Shread, 2002).

Recently we are watching a strong movement towards WSs and P2P services working in conjunction. Synergies between WS and P2P services include: 
- WS or Semantic WS discovery using a P2P-based approach (Laoveerakul, 2001), (Thaden, 2003), (Schlosser, 2002), (Banaei-Kashani, 2004)

- Peer discovery in P2P systems using WS as registries (Prasad, 2003)

- WSs interconnection in heterogeneous networks (Schattkowsky, 2004)

- Search engines, such as Google, based on P2P and WSs (Bhusate, 2003)

- JXTA projects that incorporate WSs, e.g. Chinook (Chinook, 2005)

The technologies enabling this convergence are some of the leading P2P protocols such as Jabber and JXTA, whereas Microsoft .NET Framework provides a rich platform for building P2P applications and Intel is building P2P components and services on top of Microsoft's .NET platform (Intel Press Releases, 2001).

The techniques that the P2P and Grid models use to handle some of the main issues of distributing computing are discussed in (Talia, 2003) in order to find a common foundation that could alleviate the complexities of each other and fulfill the need for secure, scalable and decentralized collaboration. Another approach to combining aspects of Grid computing with P2P architectures is found in the proposal for a new architecture stack for Grids presented in the Next Generation Grids second Group's report (NGG2, 2004).

The confluence of Web, P2P and Grid services provides the foundation for a common model - allowing applications to scale from proximity ad hoc networks to worldwide-scale distributed systems. Some approaches and research projects have started to appear towards supporting this convergence and reusability of the three categories of e-Services by providing appropriate models and platforms, e.g. for unified service discovery and composition (SODIUM, 2004) and Internet Distributed Computing (Milenkovic, 2003).

Based on the above, we propose the following roadmap actions for interparadigm interoperability:

1 Monitor progress of protocols in the e-Service area

2 Monitor integration efforts

3 Adapt interoperable protocols for synergies between different kinds of eServices, e.g. JXTA and WS standards

4 Adapt integration paradigms between different e-Services to alleviate the complexities of each other

\section{CONCLUDING SUMMARY}

The advent of Service Oriented Computing has stimulated great expectation among the developers community. However, the heterogeneity and vast amount of protocols and standards of e-Services, which many times expose overlapping applicability, hinder interoperability which is one of the basic benefits of SOA.

In this paper we investigated the interoperability potential and challenges of Web, P2P and Grid services which are the building blocks of SOA and are known as e-Services. We also presented roadmap actions both for intra- and inter-paradigm interoperability. Our observations are summarized in Table 1.

We believe that the work presented in this paper and the suggested roadmap actions can enhance interoperability in the e-Services area and help developers to take advantage of the full potential and promised benefits of Service Oriented development. 
Table 1. Interoperability approaches and roadmap actions for e-Services

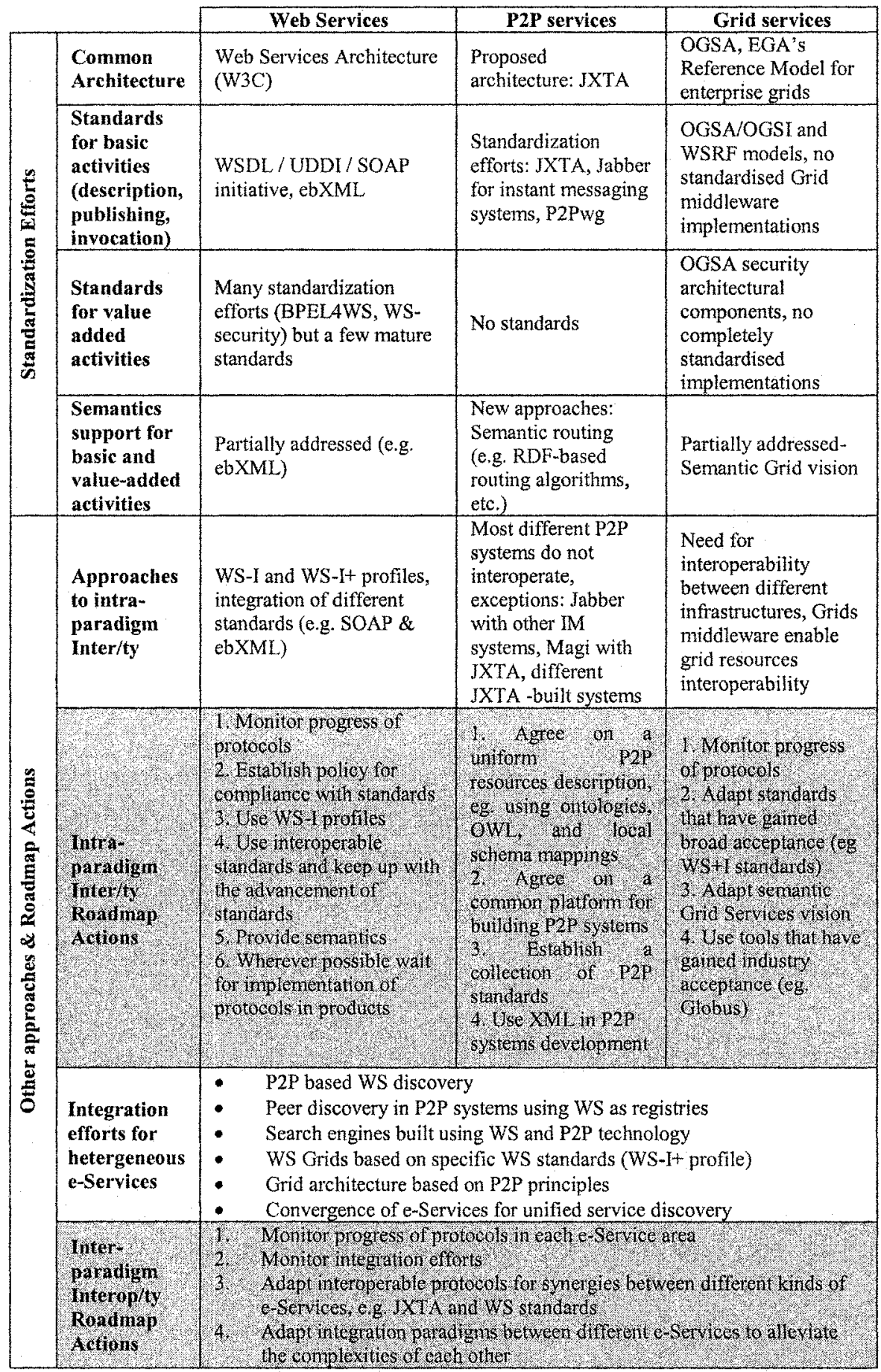




\section{ACKNOWLEDGEMENT}

Part of this work has been supported by the IST Network of Excellence INTEROP (INTEROP, 2005) and the IST Project SODIUM (SODIUM, 2004). The authors would like to thank George Athanasopoulos and Vangelis Floros for their contribution in earlier drafts of this paper.

\section{REFERENCES}

1. Aberer, K., Cudr'e-Mauroux, P.,Ouksel, A.: Emergent Semantics Principles and Issues, Proc. of 1st Int Conference on Semantics of a Networked World (ICSNW04), Paris, France, 2004

2. Atkinson, M., DeRoure, D., Dunlop, A., Fox, G., Web Service Grids: An Evolutionary Approach, 2004, UK e-Science Technical Report July 132004 to be published in a special issue of Concurrency\&Computation: Practice\&Experience 2005

3. Banaei-Kashani, F., Ching-Chien Chen, C., Shahabi, C., WSPDS: Web Services Peer-to-peer Discovery Service, Proc. of Int Symposium on Web Services and Applications (ISWS'04), 2004

4. Bhusate, A., De Meer, H., Web Services Over Infrastructure-less Networks, Proc. of the London Communications Symposium, 2003

5. Booth, D., Web Services Architecture, W3C Working Group Note, 2004, http://www.w3.org/TR/2004/NOTE-ws-arch-20040211/

6. BPELAWS 1.1, 2005, http://www-128.ibm.com/developerworks/library/specification/ws-bpel/

7. Brooke, J., Garwood, K., Goble, C.: Interoperability of Grid Resource Descriptions: A Semantic Approach, Proc. of the 1st GGF Semantic Grid Workshop, USA, 2003

8. Buehling, B.: P2P and XML in Business, published on XML.com, 2001, http://www.xml.com/pub/a/2001/07/11/xmlp2p.html

9. Chinook P2P Bioinformatics project, http://smweb.bcgsc.bc.ca/chinook.htm, last access 25/5/2005

10. ebXML, http://www.ebxml.org, last access $25 / 5 / 2005$

11. Edutella project, http://edutella.jxta.org, last access $25 / 5 / 2005$

12. EGA (Enterprise Grid Alliance), http://www.gridalliance.org/, last access 25/5/2005

13. Foster, I., Kesselman, C., Nick, J., Tuecke, S.: The Physiology of the Grid: An Open Grid Services Architecture for Distributed Systems Integration, Globus Project, 2002, http://www.globus.org/research/papers/ogsa.pdf

14. Foster, I., Kesselman, C.: The Globus Toolkit, In Ian Foster and Carl Kesselman, editors, The Grid: Blueprint for a New Computing Infrastructure, pp. 259-278. Morgan Kaufmann, San Francisco, CA, 1999, Chap. 11

15. Furmento, N., Hau, J., Lee, W., Newhouse, S., Darlington, J.: Implementations of a ServiceOriented Architecture on top of Jini, JXTA and OGSA, Proc. of the 2nd European AcrossGrids Conference, AxGrids 2004, Nicosia, Cyprus, 2004

16. GGF (Global Grid Forum), http://www.Gridforum.org/, last access 25/5/2005

17. Globus Alliance, http://www.globus.org/, last access 25/5/2005

18. Groove, http://www.groove.net, last access $25 / 5 / 2005$

19. IETF (The Internet Engineering Task Force ), http://www.ietf.org/, http://www.groove.net

20. Intel Press Releases: Intel Announces Technology Middleware To Accelerate Peer-To-Peer Capabilities, 2001, http://www.intel.com/pressroom/archive/releases/20010828tech.htm

21. INTEROP Network of Excellence, http://interop-noe.org/INTEROP/, last access 25/5/2005

22. Jabber Technology Overview, 2002, http://www.jabber.org/

23. JXTA project, http://www.jxta.org/, last access $25 / 5 / 2005$

24. Laoveerakul, S., Laongwaree, K., Tongsima, S.: Decentralized UDDI based on P2P Protocol, Proc. of P2P/NSCD session, APAN Shanghai Meetings, 2002

25. Microsoft .NET, http://www.microsoft.com/services/net/default.asp

26. Milenkovic, M., Robinson, S., Knauerhase, R., Barkai, D., Garg, S., Tewari, V., Anderson, T., Bowman, M., Intel, Toward Internet Distributed Computing, published at Computer Society (IEEE), 2003, Vol. 36, No. 5, pp. 38-46

27. Milojicic, D., Kalogeraki, V., Lukose, R., Nagaraja, K., Pruyne, J., Richard, B., Rollins, S., Xu, Z., HP Laboratories Palo Alto: Peer-to-Peer Computing, HPL-2002-57, 2003 
28. NGG2 (Next Generation Grids2nd Group) report, 2004, http:/www.cordis.lu/ist/Grids

29. OASIS, www.oasis-open.org, last access 25/5/2005

30. OGSA Glossary of Terms, GGF document GGFD-I.044, J.Treadwell, ed., Jan. 25th, 2005, http://www.ggf.org/documents/GWD-I-E/GFD-I.044.pdf

31. OGSI (Open Grid Services Interface), http:/www.Gridforum.org/Meetings/ggf7/drafts/draft-ggfogsi-Gridservice-23 2003-02-17.pdf

32. OWL Overview, 2004, http:/www.w3.org/TR/owl-features/

33. OWL-S 1.1, 2004, http://www.daml.org/services/owl-s/1.1/

34. Parastatidis, S., Webber, J., Watson, P., Rischbeck, WS-GAF: A Framework for Building Grid Applications Using Web Services, Journal of Concurrency and Computation: Practice and Experience, 17(2-4), pp. 391-417, 2005

35. P2PWG (Peer to Peer Working Group), http://P2P.internet2.edu/, last access 25/5/2005

36. Prasad, V., Lee, Y.: A Scalable Infrastructure for Peer-to-Peer Networks Using Web Services as Registries and Intelligent Peer Locators, Proc. of the 3rd International Symposium on Cluster Computing and the Grid, 2003, Tokyo, Japan

37. RDF (Resource Description Framework), http://www.w3.org/RDF/, last access 25/5/2005

38. Roure, D., Jennings, N., Shadbolt, N., The Semantic Grid: Past, Present and Future, Preprint - to appear in IEEE Proceedings March 2005

39. Schattkowsky, T., Loeser, C., Müller, W., Peer-To-Peer Technology for Interconnecting Web Services in Heterogeneous Networks, Proc. of 18th Int Conference on Advanced Information Networking and Applications (AINA'04), 2004

40. Schlosser, M., Sintek, M., Decker, S., Nejdl, W.: HyperCuP - Hypercubes, Ontologies and Efficient Search on P2P Networks, Int Workshop on Agents and Peer-to-Peer Computing, Bologna, Italy, 2002

41. Shread, P., McAfee Taps Grid Power, Web Services To Boost Security, 2002, http://www.Gridcomputingplanet.com/news/article.php/3281_1010431

42. Snelling, D., van den Berghe, S., von Laszewski, G., Wieder, P., Breuer, D., MacLaren, J., Nicole, D. and Hoppe, H. C.: A Unicore Globus Interoperability Layer, Computing and Informatics 21, pp. $399-411,2002$

43. SOAP 1.1, 2001, http://www.w3.org/TR/SOAP

44. SODIUM project, 2004, http://www.atc.gr/sodium

45. Talia, D., Trunfio, P.:Toward a Synergy Between P2P and Grids, published in IEEE Internet $\begin{array}{lllllll}\text { Computing, July/August } & \text { 2003, } & \text { Vol. } & \text { N, } & \text { No. } & \text { 4, } & \text { 94-95, }\end{array}$ http://dsonline.computer.org/0307/d/wp4P2P.htm

46. Thaden, U., Siberski, W., Nejdl, W.: A Semantic Web based Peer-to-Peer Service Registry Network, Proc. of the 1st Workshop on Semantics in Peer-to-Peer and Grid Computing, at the 12th Int Wold Wide Web Conference, 2003

47. Tsalgatidou, A., Pilioura, T.: An Overview of Standards and Related Technologyin Web Services, Internation Joumal of Distributed and Parallel Data Bases, Special Issue on E-Services 12(2), pp. $135-162,2002$

48. UDDI, http://www.uddi.org/, last access $25 / 5 / 2005$

49. W3C (World Wide Web Consortium), http://www.w3.org/, last access 25/5/2005

50. W3C Web Services Architecture Requirements document, 2002, http://www.w3.org/2002/ws/arch/2/wd-wsawg-reqs-03262002

51. Wieder, P., Rambadt, M., UNICORE - Globus: Interoperability of Grid Infrastructures, Proc. of Cray User Group Summit 2002, Manchester

52. Wilkes, L., The Web Services Protocol Stack, 2005, http://roadmap.cbdiforum.com/reports/protocols/

53. WS-CF, OASIS, 2003, http://developers.sun.com/techtopics/webservices/wscaf/wscf.pdf

54. WS-Choreography, 2002, http://www.w3.org/2002/ws/chor/

55. WS-Coordination, 2004, ftp://www6.software.ibm.com/software/developer/library/WSCoordination.pdf

56. WSDL 1.1, (Web Services Descriftp:/www6.software.ibm.com/software/developer/library/WSCoordination.pdfanguage), 2001, http://www.w3.org/TR/wsd1

57. WS-I (Web Services Interoperability Organization), http://www.ws-i.org/, last access 25/5/2005

58. WS-Notification 1.0, 2004, http://www-106.ibm.com/developerworks/library/ws-pubsub/WSPubSub.pdf

59. WSRF (The WS-Resource Framework), March 2004, http://www.globus.org/wsrf/

60. WS-Security 1.0, 2002, http://www-106.ibm.com/developerworks/webservices/library/ws-secure/ 